\title{
PARABOLIC COMPLEX MONGE-AMPÈRE TYPE EQUATIONS ON CLOSED HERMITIAN MANIFOLDS
}

\author{
WEI SUN
}

\begin{abstract}
We study the parabolic complex Monge-Ampère type equations on closed Hermitian manfolds. We derive uniform $C^{\infty}$ a priori estimates for normalized solutions, and then prove the $C^{\infty}$ convergence. The result also yields a way to carry out method of continuity for elliptic Monge-Ampére type equations.
\end{abstract}

\section{INTRODUCTION}

Let $\left(M^{n}, \omega\right)$ be a compact Hermitian manifold of complex dimension $n \geq 2$ and $\chi$ a smooth real $(1,1)$ form on $M^{n}$. Write $\omega$ and $\chi$ respectively as

$$
\omega=\frac{\sqrt{-1}}{2} \sum_{i, j} g_{i j} d z^{i} \wedge d \bar{z}^{j}
$$

and

$$
\chi=\frac{\sqrt{-1}}{2} \sum_{i, j} \chi_{i \bar{j}} d z^{i} \wedge d \bar{z}^{j} .
$$

We denote $\chi_{u}:=\chi+\frac{\sqrt{-1}}{2} \partial \bar{\partial} u$, and also set

$$
[\chi]:=\left\{\chi_{u}: u \in C^{2}(M)\right\}, \quad[\chi]^{+}:=\left\{\chi^{\prime} \in[\chi]: \chi^{\prime}>0\right\} .
$$

In this paper, we are concerned with the following equation on $M$,

$$
\frac{\partial u}{\partial t}=\ln \frac{\chi_{u}^{n}}{\chi_{u}^{n-\alpha} \wedge \omega^{\alpha}}-\ln \psi
$$

with initial value $u(x, 0)=0$. We are interested in solving the corresponding nondegenerate parabolic equations. To be parabolic, equation (1.1) must have $\chi_{u}>0$; we call such functions admissible or $\chi$-plurisubharmonic. To be nondegenerate, we need to assume $\psi>0$ on $M$.

In order to solve equation pmach-parabolic-equation, it is necessary to impose some condition. As in [17] and [21], we define, for a smooth positive real function $\psi$ on $M$,

$$
\mathscr{C}_{\alpha}(\psi):=\left\{[\chi]: \exists \chi^{\prime} \in[\chi]^{+}, n \chi_{1}^{\prime n-1}>(n-\alpha) \psi \chi^{\prime n-\alpha-1} \wedge \omega^{\alpha}\right\} .
$$


If $\chi \in \mathscr{C}_{\alpha}(\psi)$, we say that $\chi$ satisfies the cone condition.

The study of the parabolic flows is motivated by the complex Monge-Amère type equation

$$
\chi_{u}^{n}=\psi \chi_{u}^{n-\alpha} \wedge \omega^{\alpha}, \chi_{u}>0 .
$$

A critical point of the flow gives a Hermitian metric $\chi$ satisfying

$$
\chi_{u}^{n}=e^{b} \psi \chi_{u}^{n-\alpha} \wedge \omega^{\alpha}, \chi_{u}>0
$$

for some real constant $b$.

When $\alpha=n$, it is exactly the complex Monge-Ampère equation, which is strongly connected with complex geometry. In the fundamental work of Yau [27] (see also [1]), he proved the Calabi conjecture [3], [4] by solving the complex Monge-Ampère equation. Cao [5] reproduced the result of Yau [27] and Aubin [1] by Kähler-Ricci flow. Cherrier [8], Tosatti and Weinkove [23] independently extended the zero order estimate of Yau [27] to Hermitian manifolds under the balanced condition, and then solved the complex Monge-Ampère equations on closed Hermitian manifolds by method of continuity. Later, Tosatti and Weinkove [24] successfully removed the balanced condition and extended the result to general Hermitian manifolds. Gill [15] introduced the Chern-Ricci flow, and gave a parabolic proof for the result in [24].

For $\alpha=1$, equation (1.3) was proposed by Donaldson [9] in connection with moment maps and is closely related to the Mabuchi energy [7], [26], [20] for Kähler manifolds. It is well known that when $\chi$ and $\omega$ are Kähler, there is an invariant defined by

$$
c=\frac{\int_{M} \chi^{n}}{\int_{M} \chi^{n-\alpha} \wedge \omega^{\alpha}} .
$$

The equation was studied by Chen [6], [7], Weinkove [25], [26], Song and Weinkove [20] using the $J$-flow where $\psi=c$. Their result was extended by Fang, Lai and Ma [11] to all $1 \leq \alpha<n$. In [12], [13], Fang and Lai studied a class of geometric flows when $\psi=c$, which include equation (1.1). It attracts our attention to generalize the results to general Hermitian manifolds or general $\psi$. Guan and the author [17] studied the Dirichlet problem on general Hermitian manifolds with the assumption of subsolution. The author [21] solved equation (1.3) by method of continuity on closed Hermitian manifolds under the cone condition.

In the study, the sharp $C^{2}$ estimate is the key. We prove the following theorem. 
Theorem 1.1. Let $\left(M^{n}, \omega\right)$ be a closed Hermitian manifold of complex dimension $n$. Suppose that $\chi$ is a smooth real $(1,1)$ form satisfying $\chi \in \mathscr{C}_{\alpha}(\psi)$. Then there exists a long time solution $u$ to equation (1.1). Moreover, there are constants $C$ and $A$ such that

$$
\Delta u+\operatorname{tr} \chi \leq C e^{A\left(u-\inf _{M \times[0, t]} u\right)}
$$

where $C, A$ depend only on initial geometric data.

The $C^{2}$ estimate is much more improved than that in [22], which is

$$
\Delta u+\operatorname{tr} \chi C e^{\left(e^{A\left(\sup _{M \times[0, t]}(u-\underline{u})-\inf _{M \times[0, t]}(u-\underline{u})\right)-e^{A\left(\sup _{M \times[0, t]}(u-\underline{u})-(u-\underline{u})\right)}}\right)} .
$$

The improved $C^{2}$ estimate can help us to obtain the uniform a priori $C^{0}$ estimate if we have proper conditions. The $C^{0}$ is one of the most difficult estimates for differential equations on closed manifolds. In fact, the higher order estimates thus follow from the $C^{0}$ and $C^{2}$ estimates, Evans-Krylov theory [10], [18] and Schauder estimates, which are quite standard procedures.

We do not impose strong condition on $\psi$, and consequently it is very likely that the flow $u$ itself does not converge. To discover some convergence property, it is necessary to normalize the solution. Let

$$
\tilde{u}=u-\frac{\int_{M} u \omega^{n}}{\int \omega^{n}} .
$$

For general Hermitian manifolds, we have the following result.

Theorem 1.2. Under the assumption of Theorem 1.1, there exists a uniform constant $C$ such that for all time $t \geq 0$,

$$
\sup _{x \in M} u(x, t)-\inf _{x \in M} u(x, t)<C,
$$

given that

$$
\frac{\chi^{n}}{\chi^{n-\alpha} \wedge \omega^{\alpha}} \leq \psi
$$

Then $\tilde{u}$ is $C^{\infty}$ convergent to a smooth function $\tilde{u}_{\infty}$. Moreover, there is a unique real number $b$ such that the pair $\left(\tilde{u}_{\infty}, b\right)$ solves equation (1.4).

Should we have more knowledge of the manifold, it would be possible to obtain deeper results. When $\chi$ and $\omega$ are both Kähler, we are able to solve Donaldson's 
problem formerly proven by the flow method in [20], [11]. It is worth a mention that the former results are only for the special case $\psi=c$ while we only require that $\psi \geq c$.

It is noticeable that in [21], the elliptic approach has no obstacle when $\psi \geq c$ while the former parabolic approaches have difficulties to treat non-constant $\psi$. It is natural to ask: is the flow method also able to treat $\psi \geq c$ ? After a study of $J$-functional [6], we use the functional to normalize the velocity $\partial_{t} u$ instead of the function $u$. The idea is quite simple and natural: we pull back the surface at every instantaneous moment.

Theorem 1.3. Let $\left(M^{n}, \omega\right)$ be a closed Kähler manifold of complex dimension $n$ and $\chi$ is also Kähler. Suppose that $\chi \in \mathscr{C}_{\alpha}(\psi)$ and $\psi \geq c$ for all $x \in M$, where $c$ is defined in (1.5). Then there exists a uniform constant $C$ such that for all time $t \geq 0$,

$$
\sup _{x \in M} u(x, t)-\inf _{x \in M} u(x, t)<C \text {. }
$$

Consequently, $\tilde{u}$ is $C^{\infty}$ convergent to a smooth function $\tilde{u}_{\infty}$. Moreover, there is a unique real number $b$ such that the pair $\left(\tilde{u}_{\infty}, b\right)$ solves equation (1.4).

We organize the paper as follows. In section 2, we state and show some preliminary knowledge related to equation (1.1). In section 3, we establish the improved $C^{2}$ estimate. In section 4 , we study the long time existence of the solution flow. The $C^{2}$ estimate proven in section 3 helps us to obtain the uniform $C^{0}$ estimate, and hence we show that higher order estimates are also uniform. In section 5 , we give a proof for a Li-Yau type Harnack inequality. In section 6, we apply the inequality to show that the time derivative of $\tilde{u}$ decays exponentially. As an immediate result, we show that $\tilde{u}$ convergences to a smooth function $\tilde{u}_{\infty}$, which solves equation (1.4) for some $b$. In section 7 , we apply Theorem 1.2 to carrying out method of continuity, in order to avoid using some specific knowledge.

\section{Preliminaries}

We shall follow [17] for notations. In particular, $g$ and $\nabla$ will denote the Riemannian metric and the corresponding Chern connection of $(M, \omega)$. In local coordinates $z=$ 
$\left(z^{1}, \cdots, z^{n}\right)$ we have

$$
\left\{\begin{array}{c}
T_{i j}^{k}=\sum_{l} g^{k \bar{l}}\left(\frac{\partial g_{j \bar{l}}}{\partial z^{i}}-\frac{\partial g_{i \bar{l}}}{\partial z^{j}}\right) \\
R_{i \bar{j} k \bar{l}}=-\frac{\partial^{2} g_{k \bar{l}}}{\partial z^{i} \partial \bar{z}^{j}}+\sum_{p, q} g^{p \bar{q}} \frac{\partial g_{k \bar{q}}}{\partial z^{i}} \frac{\partial g_{p \bar{l}}}{\partial \bar{z}^{j}}
\end{array}\right.
$$

For convenince, we set

$$
X:=\chi_{u}=\chi+\frac{\sqrt{-1}}{2} \partial \bar{\partial} u
$$

and thus

$$
X_{i \bar{j}}=\chi_{i \bar{j}}+\bar{\partial}_{j} \partial_{i} u \text {. }
$$

Also, we denote the coefficients of $X^{-1}$ by $X^{i \bar{j}}$. It is easy to see that

$$
\overline{X_{i \bar{j} k}}=X_{j \bar{i} \bar{k}} \text {. }
$$

Assuming at the point $p, g_{i \bar{j}}=g_{i j}$ and $X_{i \bar{j}}$ is diagonal in a specific chart. Therefore,

$$
\begin{gathered}
X_{i \bar{i} \bar{j} \bar{j}}-X_{j \bar{j} \bar{i} \bar{i}}=R_{j \bar{j} \bar{i} \bar{i}} X_{i \bar{i}}-R_{i \bar{i} \bar{j} \bar{j}} X_{j \bar{j}}+2 \mathfrak{R e}\left\{\sum_{p} \overline{T_{i j}^{p}} X_{i \bar{p} j}\right\} \\
-\sum_{p} T_{i j}^{p} \overline{T_{i j}^{p}} X_{p \bar{p}}-G_{i \bar{i} \bar{j} \bar{j}}
\end{gathered}
$$

where

$$
\begin{aligned}
G_{i \bar{i} j \bar{j}}=\chi_{j \bar{j} i \bar{i}} & -\chi_{i \bar{i} j \bar{j}}+\sum_{p} R_{j \bar{j} i \bar{p}} \chi_{p \bar{i}}-\sum_{p} R_{i \bar{i} j \bar{p}} \chi_{p \bar{j}} \\
& +2 \mathfrak{R e}\left\{\sum_{p} \overline{T_{i j}^{p}} \chi_{i \bar{p} j}\right\}-\sum_{p, q} T_{i j}^{p} \overline{T_{i j}^{q}} \chi_{p \bar{q}} .
\end{aligned}
$$

Let $S_{\alpha}(\lambda)$ denote the $\alpha$-th elementary symmetric polynomial of $\lambda \in \mathbb{R}^{n}$,

$$
S_{\alpha}(\lambda)=\sum_{1 \leq i_{1}<\cdots<i_{\alpha} \leq n} \lambda_{i_{1}} \cdots \lambda_{i_{\alpha}} .
$$

For a square matrix $A$, define $S_{\alpha}(A)=S_{\alpha}(\lambda(A))$ where $\lambda(A)$ denote the eigenvalues of $A$. Further, write $S_{\alpha}(X)=S_{\alpha}\left(\lambda_{*}(X)\right)$ and $S_{\alpha}\left(X^{-1}\right)=S_{\alpha}\left(\lambda^{*}(X)\right)$ where $\lambda_{*}(X)$ and $\lambda^{*}(X)$ denote the eigenvalues of a Hermitian matrix $X$ with respect to $\omega$ and to $\omega^{-1}$, respectively. Unless otherwise indicated we shall use $S_{\alpha}$ to denote $S_{\alpha}\left(X^{-1}\right)$ when no possible confusion would occur. 
In local coordinates, we can write equation (1.1) in the form

$$
\frac{\partial u}{\partial t}=\ln S_{n}\left(\chi_{u}\right)-\ln S_{n-\alpha}\left(\chi_{u}\right)+\ln C_{n}^{\alpha}-\ln \psi .
$$

or equivalently,

$$
\frac{\partial u}{\partial t}=\ln C_{n}^{\alpha}-\ln \psi-\ln S_{\alpha}\left(\chi_{u}^{-1}\right)
$$

Differentiating this equation twice at $p$ and applying the strong concavity of $S_{\alpha}$ as in [17], we have

$$
\partial_{t} \partial_{l} u=-\frac{\partial_{l} \psi}{\psi}+S_{\alpha}^{-1} \sum_{i} S_{\alpha-1 ; i}\left(X^{i \bar{i}}\right)^{2} X_{i \bar{i} l}
$$

and

$$
\begin{gathered}
\partial_{t} \bar{\partial}_{l} \partial_{l} u \leq C+S_{\alpha}^{-2} \sum_{i} \partial_{i} S_{\alpha} \bar{\partial}_{i} S_{\alpha}-S_{\alpha}^{-1} \sum_{i, j} S_{\alpha-1 ; i}\left(X^{i \bar{i}}\right)^{2} X^{j \bar{j}} X_{j \bar{i} \bar{l}} X_{i \bar{j} l} \\
+S_{\alpha}^{-1} \sum_{i} S_{\alpha-1 ; i}\left(X^{i \bar{i}}\right)^{2} X_{i \bar{i} l \bar{l}}
\end{gathered}
$$

Since $\underline{u} \in C^{2}(M)$ and $\chi_{\underline{u}}>0$,

$$
\epsilon \omega \leq \chi_{\underline{u}} \leq \epsilon^{-1} \omega
$$

for some $\epsilon>0$.

Here we write down an alternative statement, which will be used later. It is a key theorem in [11].

Theorem 2.1. We have an alternative

$$
\sum_{i} S_{\alpha-1 ; i}\left(X^{i \bar{i}}\right)^{2}\left(\chi_{i \bar{i}}+\bar{\partial}_{i} \partial_{i} \underline{u}\right) \geq(1+\theta) \alpha\left(\frac{\psi}{C_{n}^{\alpha}}\right)^{1 / \alpha} S_{\alpha}^{1+1 / \alpha}
$$

for some $\theta>0$, or

$$
w \leq C
$$

for some $C>0$ large enough and independent on $u$.

Moreover, by the maximum principle, $\frac{\partial u}{\partial t}$ reaches the extremal values at $t=0$, that is, on a maximal time interval $[0, T)$,

$$
\left|\frac{\partial u}{\partial t}\right| \leq \sup _{M \times\{0\}}\left|\frac{\partial u}{\partial t}\right| .
$$


As an immediate corollary,

$$
\left|\frac{\partial \tilde{u}}{\partial t}\right| \leq 2 \sup _{M \times\{0\}}\left|\frac{\partial u}{\partial t}\right|
$$

and

$$
\left|\ln \frac{\chi_{u}^{n}}{\chi_{u}^{n-\alpha} \wedge \omega^{\alpha}}-\ln \psi\right| \leq \sup _{M \times\{0\}}\left|\ln \frac{\chi_{u}^{n}}{\chi_{u}^{n-\alpha} \wedge \omega^{\alpha}}-\ln \psi\right| .
$$

Therefore, the flow remains Hermitian at any time.

\section{THE SECOND ORDER ESTIMATES}

In this section we derive the second order estimate for admissible solutions.

Proposition 3.1. Let $u \in C^{4}(M \times[0, T))$ be an admissible solution of equation (1.1) and $w=\Delta u+\operatorname{tr} \chi$. Then there are uniform positive constants $C$ and $A$ such that

$$
w \leq C e^{A\left(u-\inf _{M \times[0, t]} u\right)},
$$

where $C, A$ depend only on geometric data.

Proof. Consider the function $e^{\phi} w$ where $\phi$ is to be specified later. Suppose that $e^{\phi} w$ its maximum at some point $\left(p, t_{0}\right) \in M_{t}=M \times(0, t]$. Choose a local chart near $p$ such that $g_{i \bar{j}}=\delta_{i j}$ and $X_{i \bar{j}}$ is diagonal at $p$ when $t=t_{0}$. Therefore, we have at the point $\left(p, t_{0}\right)$

$$
\begin{gathered}
\frac{\partial_{l} w}{w}+\partial_{l} \phi=0, \\
\frac{\bar{\partial}_{l} w}{w}+\bar{\partial}_{l} \phi=0, \\
\frac{\partial_{t} w}{w}+\partial_{t} \phi \geq 0
\end{gathered}
$$

and

$$
\frac{\bar{\partial}_{l} \partial_{l} w}{w}-\frac{\bar{\partial}_{l} w \partial_{l} w}{w^{2}}+\bar{\partial}_{l} \partial_{l} \phi \leq 0 .
$$


Applying $\frac{\partial}{\partial t}-S_{\alpha}^{-1} \sum_{l} S_{\alpha-1 ; l}\left(X^{l \bar{l}}\right)^{2} \partial_{l} \bar{\partial}_{l}$ to the function $e^{\phi} w$,

$$
\begin{aligned}
& \partial_{t}\left(e^{\phi} w\right)-S_{\alpha}^{-1} \sum_{l} S_{\alpha-1 ; l}\left(X^{l \bar{l}}\right)^{2} \partial_{l} \bar{\partial}_{l}\left(e^{\phi} w\right) \\
= & e^{\phi}\left(w \partial_{t} \phi+\partial_{t} w-S_{\alpha}^{-1} \sum_{l} S_{\alpha-1 ; l}\left(X^{l \bar{l}}\right)^{2}\left(w \partial_{l} \bar{\partial}_{l} \phi-w^{-1}\left|\partial_{l} w\right|^{2}+\partial_{l} \bar{\partial}_{l} w\right)\right) .
\end{aligned}
$$

This formula, with (3.4) and (3.5), leads to that at $\left(p, t_{0}\right)$

$$
0 \leq w \partial_{t} \phi+\partial_{t} w-S_{\alpha}^{-1} \sum_{l} S_{\alpha-1 ; l}\left(X^{l \bar{l}}\right)^{2}\left(w \bar{\partial}_{l} \partial_{l} \phi-w^{-1}\left|\partial_{l} w\right|^{2}+\sum_{i} X_{i \bar{i} l \bar{l}}\right) .
$$

By (2.5) and (2.11), we have at $\left(p, t_{0}\right)$

$$
\begin{aligned}
& \quad \sum_{l} S_{\alpha-1 ; l}\left(X^{l \bar{l}}\right)^{2}\left(w \bar{\partial}_{l} \partial_{l} \phi-w^{-1}\left|\partial_{l} w\right|^{2}+\sum_{i} X_{i \bar{i} l \bar{l}}\right) \\
& \geq w \sum_{i} S_{\alpha-1 ; i}\left(X^{i \bar{i}}\right)^{2} \bar{\partial}_{i} \partial_{i} \phi-w^{-1} \sum_{i} S_{\alpha-1 ; i}\left(X^{i \bar{i}}\right)^{2}\left|\partial_{i} w\right|^{2} \\
& \quad+\sum_{i, j} S_{\alpha-1 ; i}\left(X^{i \bar{i}}\right)^{2}\left(-R_{j \bar{j} i \bar{i}} X_{i \bar{i}}+R_{i \bar{i} j \bar{j}} X_{j \bar{j}}+G_{i \bar{i} j \bar{j}}\right) \\
& \quad-2 \sum_{i, j, l} S_{\alpha-1 ; i}\left(X^{i \bar{i}}\right)^{2} \mathfrak{R e}\left\{\overline{T_{i l}^{j}} X_{i \bar{j} l}\right\}+\sum_{i, j, l} S_{\alpha-1 ; i}\left(X^{i \bar{i}}\right)^{2} T_{i l}^{j} \overline{T_{i l}^{j}} X_{j \bar{j}} \\
& \quad+\sum_{i, j, l} S_{\alpha-1 ; i}\left(X^{i \bar{i}}\right)^{2} X^{j \bar{j}} X_{j \bar{i} \bar{l}} X_{i \bar{j} l}+S_{\alpha} \sum_{i} \partial_{t} \bar{\partial}_{i} \partial_{i} u-C S_{\alpha} .
\end{aligned}
$$

As in [21], we can simplify equation (3.8),

$$
\begin{aligned}
& \sum_{l} S_{\alpha-1 ; l}\left(X^{l \bar{l}}\right)^{2}\left(w \bar{\partial}_{l} \partial_{l} \phi-w^{-1}\left|\partial_{l} w\right|^{2}+\sum_{i} X_{i \bar{i} l \bar{l}}\right) \\
\geq & S_{\alpha} \sum_{i} \partial_{t} \bar{\partial}_{i} \partial_{i} u-C S_{\alpha}+\sum_{i, j} S_{\alpha-1 ; i}\left(X^{i \bar{i}}\right)^{2}\left(-R_{j \bar{j} i \bar{i}} X_{i \bar{i}}+R_{i \bar{i} \bar{j} \bar{j}} X_{j \bar{j}}+G_{i \bar{i} \bar{j} \bar{j}}\right) \\
& +w \sum_{i} S_{\alpha-1 ; i}\left(X^{i \bar{i}}\right)^{2} \bar{\partial}_{i} \partial_{i} \phi+\frac{2}{w} \sum_{i, j} S_{\alpha-1 ; i}\left(X^{i \bar{i}}\right)^{2} \mathfrak{R e}\left\{\hat{T}_{j i}^{k} \chi_{k \bar{j}} \bar{\partial}_{i} w\right\}
\end{aligned}
$$

where $\hat{T}$ denotes the torsion with respect to the Hermitian metric $\chi$. In [21], we just simply threw away the last term.

Now we apply a trick due to Phong and Sturm [19] and use the following function

$$
\phi:=-A(u-\underline{u})+\frac{1}{u-\underline{u}-\inf _{M_{t}}(u-\underline{u})+1}=-A(u-\underline{u})+E_{1} .
$$


Without loss of genelarity, we can assume $A \gg 1$ throughout this paper.

It is easy to see that

$$
\begin{gathered}
\partial_{t} \phi=-A \partial_{t} u-\partial_{t} u E_{1}^{2}, \\
\partial_{i} \phi=-A\left(\partial_{i} u-\partial_{i} \underline{u}\right)-\left(\partial_{i} u-\partial_{i} \underline{u}\right) E_{1}^{2}
\end{gathered}
$$

and

$$
\bar{\partial}_{i} \partial_{i} \phi=-A\left(\bar{\partial}_{i} \partial_{i} u-\bar{\partial}_{i} \partial_{i} \underline{u}\right)-\left(\bar{\partial}_{i} \partial_{i} u-\bar{\partial}_{i} \partial_{i} \underline{u}\right) E_{1}^{2}+2\left|\partial_{i}(u-\underline{u})\right|^{2} E_{1}^{3} .
$$

The fourth term in (3.9) turns to

$$
\begin{aligned}
w \sum_{i} S_{\alpha-1 ; i}\left(X^{i \bar{i}}\right)^{2} \bar{\partial}_{i} \partial_{i} \phi= & 2 w \sum_{i} S_{\alpha-1 ; i}\left(X^{i \bar{i}}\right)^{2}\left|\partial_{i}(u-\underline{u})\right|^{2} E_{1}^{3} \\
& -\left(A w+w E_{1}^{2}\right) \sum_{i} S_{\alpha-1 ; i}\left(X^{i \bar{i}}\right)^{2}\left(\bar{\partial}_{i} \partial_{i} u-\bar{\partial}_{i} \partial_{i} \underline{u}\right) ;
\end{aligned}
$$

and the fifth term is

$$
\begin{array}{r}
\frac{2}{w} \sum_{i, j} S_{\alpha-1 ; i}\left(X^{i \bar{i}}\right)^{2} \mathfrak{R e}\left\{\hat{T}_{j i}^{k} \chi_{k \bar{j}} \bar{\partial}_{i} w\right\}=-2 \sum_{i, j} S_{\alpha-1 ; i}\left(X^{i \bar{i}}\right)^{2} \mathfrak{R e}\left\{\hat{T}_{j i}^{k} \chi_{k \bar{j}} \bar{\partial}_{i} \phi\right\} \\
\geq-w E_{1}^{3} \sum_{i} S_{\alpha-1 ; i}\left(X^{i \bar{i}}\right)^{2}\left|\partial_{i}(u-\underline{u})\right|^{2}-\frac{C A^{2}}{w E_{1}^{3}} \sum_{i} S_{\alpha-1 ; i}\left(X^{i \bar{i}}\right)^{2} .
\end{array}
$$

Therefore,

$$
\begin{aligned}
& S_{\alpha}^{-1} \sum_{l} S_{\alpha-1 ; l}\left(X^{l \bar{l}}\right)^{2}\left(w \bar{\partial}_{l} \partial_{l} \phi-w^{-1}\left|\partial_{l} w\right|^{2}+\sum_{i} X_{i \bar{i} l \bar{l}}\right) \\
\geq & \left(A+E_{1}^{2}\right) w S_{\alpha}^{-1} \sum_{i} S_{\alpha-1 ; i}\left(X^{i \bar{i}}\right)^{2}\left(\bar{\partial}_{i} \partial_{i} \underline{u}-\bar{\partial}_{i} \partial_{i} u\right)-\frac{C A^{2}}{w E_{1}^{3}} S_{\alpha}^{-1} \sum_{i} S_{\alpha-1 ; i}\left(X^{i \bar{i}}\right)^{2} \\
& +S_{\alpha}^{-1} \sum_{i, j} S_{\alpha-1 ; i}\left(X^{i \bar{i}}\right)^{2}\left(-R_{j \bar{j} i \bar{i}} X_{i \bar{i}}+R_{i \bar{i} \bar{j} \bar{j}} X_{j \bar{j}}+G_{i \bar{i} \bar{j} \bar{j}}\right)+\sum_{i} \partial_{t} \bar{\partial}_{i} \partial_{i} u-C .
\end{aligned}
$$

Note also that

$$
w \partial_{t} \phi+\partial_{t} w=-\left(A+E_{1}^{2}\right) w \ln \left(\frac{C_{n}^{\alpha}}{\psi S_{\alpha}}\right)+\sum_{i} \bar{\partial}_{i} \partial_{i} \partial_{t} u
$$


Combing (3.7), (3.16) and (3.17),

$$
\begin{aligned}
0 \leq & -\left(A+E_{1}^{2}\right) w\left(\ln \left(\frac{C_{n}^{\alpha}}{\psi S_{\alpha}}\right)+S_{\alpha}^{-1} \sum_{i} S_{\alpha-1 ; i}\left(X^{i \bar{i}}\right)^{2}\left(\bar{\partial}_{i} \partial_{i} \underline{u}-\bar{\partial}_{i} \partial_{i} u\right)\right) \\
& +\frac{C A^{2}}{w E_{1}^{3}} S_{\alpha}^{-1} \sum_{i} S_{\alpha-1 ; i}\left(X^{i \bar{i}}\right)^{2}+C(w+1) S_{\alpha}^{-1} \sum_{i, j} S_{\alpha-1 ; i}\left(X^{i \bar{i}}\right)^{2}+C .
\end{aligned}
$$

For $A \gg 1$ which is to be determined later, there are two cases in consideration: (1) $w>A\left(u-\underline{u}-\inf _{M_{t}}(u-\underline{u})+1\right)^{\frac{3}{2}} \geq A>N$, where $N$ is the crucial constant in Therorem 2.1; $(2) w \leq A\left(u-\underline{u}-\inf _{M_{t}}(u-\underline{u})+1\right)^{\frac{3}{2}}$.

In the first case, by Theorem 2.1,

$$
\begin{aligned}
& \ln \left(\frac{C_{n}^{\alpha}}{\psi S_{\alpha}}\right)+S_{\alpha}^{-1} \sum_{i} S_{\alpha-1 ; i}\left(X^{i \bar{i}}\right)^{2}\left(\bar{\partial}_{i} \partial_{i} \underline{u}-\bar{\partial}_{i} \partial_{i} u\right) \\
\geq & \ln \left(\frac{C_{n}^{\alpha}}{\psi S_{\alpha}}\right)+(1+\theta) \alpha\left(\frac{\psi S_{\alpha}}{C_{n}^{\alpha}}\right)^{\frac{1}{\alpha}}-\alpha \\
\geq & \theta \alpha\left(\frac{\psi S_{\alpha}}{C_{n}^{\alpha}}\right)^{\frac{1}{\alpha}}
\end{aligned}
$$

by observing the simple fact

$$
\ln x \leq x-1, \quad \forall x>0 .
$$

Alloting the extra positive $\theta$ properly, we have for $\delta>0$ small enough,

$$
0 \leq-A w \delta\left(1+S_{\alpha}^{-1} \sum_{i} S_{\alpha-1 ; i}\left(X^{i \bar{i}}\right)^{2}\right)+C w S_{\alpha}^{-1} \sum_{i, j} S_{\alpha-1 ; i}\left(X^{i \bar{i}}\right)^{2}+C .
$$

This gives a bound $w \leq 1$ at $p$ if we pick a sufficiently large $A$, which contradicts the assumption $A \gg 1$.

In the second case,

$$
\begin{aligned}
w e^{\phi} \leq\left. w e^{\phi}\right|_{p} & \leq A\left(u-\underline{u}-\inf _{M_{t}}(u-\underline{u})+1\right)^{\frac{3}{2}} e^{-A(u-\underline{u})+1} \\
& \leq A e^{2} e^{-A \inf _{M_{t}}(u-\underline{u})}
\end{aligned}
$$

and hence

$$
\begin{aligned}
w & \leq A e^{2} e^{A(u-\underline{u})-E_{1}-A \inf _{M_{t}}(u-\underline{u})} \\
& \leq A e^{2} e^{A(u-\underline{u})-A \inf _{M_{t}}(u-\underline{u})} \leq C e^{A\left(u-\inf _{M_{t}} u\right)} .
\end{aligned}
$$


The Evans-Krylov theory and Schauder estimates are quite standard, so we omit the proofs here. We refer readers to [16], [23], [21], [15] and [22].

\section{LONG TIME EXISTENCE AND UNIFORM ESTIMATES}

Since $\frac{\partial u}{\partial t}$ is bounded, in finite time we have $C^{0}$ estimate dependent on time $t$. By the Evans-Krylov theory and Schauder estimates, we can obtain time-dependent $C^{\infty}$ estimates. With a standard argument, theses estimates are sufficient for us to prove the long time existence.

Nevertheless, in order to prove the convergence, we need uniform $C^{0}$ estimate, which is independent from the time $t$.

Theorem 4.1. Under the assumption of Theorem 1.1, there exists a uniform constant $C$ depeding only on the initial geometric data such that

$$
\sup _{M} u(x, t)-\inf _{M} u(x, t) \leq C,
$$

given that

$$
\frac{\chi^{n}}{\chi^{n-\alpha} \wedge \omega^{\alpha}} \leq \psi .
$$

Proof. Following [26], we prove the theorem by contradiction. If such a bound does not exist, we can choose a sequence of time points $t_{i} \rightarrow \infty$ such that

$$
\sup _{M} u\left(x, t_{i}\right)-\inf _{M} u\left(x, t_{i}\right) \rightarrow \infty,
$$

Note that

$$
\frac{\partial u}{\partial t} \leq 0
$$

by (4.2) and the maximum principle. Thus for $t>s \geq 0$,

$$
\sup _{M} u(x, t) \leq \sup _{M} u(x, s)
$$

and

$$
\inf _{M} u(x, t) \leq \inf _{M} u(x, s) .
$$

So

$$
\inf _{M} u\left(x, t_{i}\right)=\inf _{t \in\left[0, t_{i}\right]} \inf _{M} u(x, t) \rightarrow-\infty .
$$


By Proposition 3.1,

$$
\begin{aligned}
w\left(x, t_{i}\right) & \leq C e^{A\left(u\left(x, t_{i}\right)-\inf _{M \times\left[0, t_{i}\right]} u\right)} \\
& =C e^{A\left(u\left(x, t_{i}\right)-\inf _{M} u\left(x, t_{i}\right)\right)} .
\end{aligned}
$$

As shown in [23], the sharp $C^{2}$ estimate implies that

$$
\sup _{M} u\left(x, t_{i}\right)-\inf _{M} u\left(x, t_{i}\right) \leq C,
$$

for some positive constant $C$, which is a contradiction.

As a consequence, we also obtain uniform the $C^{2}$ estimate. By (4.6), it must be

$$
w(x, t) \leq C e^{A\left(u(x, t)-\inf _{M} u(x, t)\right)} \leq C e^{A\left(\sup _{M} u(x, t)-\inf _{M} u(x, t)\right)} .
$$

Should we have more knowledge of the two metrics, there would be chances to obtain deeper results.

Theorem 4.2. Under the assumption of Theorem 1.3, there exists a uniform constant $C$ depeding only on the initial geometric data such that

$$
\sup _{M} u(x, t)-\inf _{M} u(x, t) \leq C,
$$

given that

$$
\psi \geq c
$$

where $c$ is a well known invariant defined by (1.5).

First of all, we need to extend the definition of $J$-functional [6] , which was done in [11]. Let $\mathcal{H}$ be the space of Kähler potentials

$$
\mathcal{H}:=\left\{u \in C^{\infty}(M) \mid \chi_{u} \in[\chi]^{+}\right\} .
$$

For any curve $v(s) \in \mathcal{H}$, we define the funtional $J_{\alpha}$ by

$$
\frac{d J_{\alpha}}{d s}=\int_{M} \frac{\partial v}{\partial s} \chi_{v}^{n-\alpha} \wedge \omega^{\alpha} .
$$

Then we have a formula for $J_{\alpha}$ of function $u$,

$$
J_{\alpha}(u)=\int_{0}^{1} \int_{M} \frac{\partial v}{\partial s} \chi_{v}^{n-\alpha} \wedge \omega^{\alpha} d s
$$


where $v(s)$ is an arbitrary path in $\mathcal{H}$ connecting 0 and $u$. It is straighforward to verify that the functional is independent on the path. So we can restrict the integration to the straight line $v(s)=s u$ to calculate $J_{\alpha}(u)$ for any $u \in \mathcal{H}$.

$$
\begin{aligned}
J_{\alpha}(u) & =\int_{0}^{1} \int_{M} u \chi_{s u}^{n-\alpha} \wedge \omega^{\alpha} d s \\
& =\int_{0}^{1} \int_{M} u\left(s \chi_{u}+(1-s) \chi\right)^{n-\alpha} \wedge \omega^{\alpha} d s \\
& =\int_{0}^{1} \int_{M} u \sum_{i=0}^{n-\alpha} C_{n-\alpha}^{i} s^{i}(1-s)^{n-\alpha-i} \chi_{u}^{i} \wedge \chi^{n-\alpha-i} \wedge \omega^{\alpha} d s \\
& =\sum_{i=0}^{n-\alpha} C_{n-\alpha}^{i} \int_{0}^{1} s^{i}(1-s)^{n-\alpha-i} d s \int_{M} u \chi_{u}^{i} \wedge \chi^{n-\alpha-i} \omega^{\alpha} \\
& =\frac{1}{n-\alpha+1} \sum_{i=0}^{n-\alpha} \int_{M} u \chi_{u}^{i} \wedge \chi^{n-\alpha-i} \omega^{\alpha} .
\end{aligned}
$$

Also, along the solution flow $u(x, t)$ to equation (1.1),

$$
\begin{aligned}
\frac{d}{d t} J_{\alpha}(u) & =\int_{M} \frac{\partial u}{\partial t} \chi_{u}^{n-\alpha} \wedge \omega^{\alpha} \\
& \leq \int_{M}\left(\ln \frac{\chi_{u}^{n}}{\chi_{u}^{n-\alpha} \wedge \omega^{\alpha}}-\ln \psi\right) \chi_{u}^{n-\alpha} \wedge \omega^{\alpha} \\
& \leq \ln c \int_{M} \chi_{u}^{n-\alpha} \wedge \omega^{\alpha}-\int_{M} \ln \psi \chi_{u}^{n-\alpha} \wedge \omega^{\alpha} \\
& \leq 0 .
\end{aligned}
$$

The second inequality follows from Jesen's inequality. Thus, $J_{\alpha}(u)$ is decreasing and nonpositive along the solution flow.

Now we consider an arbitrary function flow $u(x, t)$ starting from 0 . Computing $J_{\alpha}$ on the flow, that is $v(x, s)=u(x, s T)$, it follows that

$$
\begin{aligned}
J_{\alpha}(u(T)) & =\int_{0}^{1} \int_{M} T \frac{\partial u}{\partial t}(s T) \chi_{u(s T)}^{n-\alpha} \wedge \omega^{\alpha} d s \\
& =\int_{0}^{T} \int_{M} \frac{\partial u}{\partial t} \chi_{u}^{n-\alpha} \wedge \omega^{\alpha} d t \\
& =\int_{0}^{T} \frac{d J_{\alpha}}{d t} d t .
\end{aligned}
$$


For the solution flow $u(x, t)$ to equation (1.1), let

$$
\hat{u}=u-\frac{J_{\alpha}(u)}{\int_{M} \chi^{n-\alpha} \wedge \omega^{\alpha}} .
$$

\section{Lemma 4.3.}

$$
0 \leq \sup _{M} \hat{u}(x, t) \leq-C_{1} \inf _{M} \hat{u}(x, t)+C_{2} .
$$

Proof. We shall use the functional $J_{\alpha}$. By (4.18),

$$
\begin{aligned}
J_{\alpha}(\hat{u}(T)) & =\int_{0}^{T} \int_{M} \frac{\partial \hat{u}}{\partial t} \chi_{u}^{n-\alpha} \wedge \omega^{\alpha} d t \\
& =\int_{0}^{T} \int_{M}\left(\frac{\partial u}{\partial t}-\frac{1}{\int_{M} \chi^{n-\alpha} \wedge \omega^{\alpha}} \frac{d J_{\alpha}}{d t}\right) \chi_{u}^{n-\alpha} \wedge \omega^{\alpha} d t \\
& =0
\end{aligned}
$$

But according to (4.16), we have

$$
\frac{1}{n-\alpha+1} \sum_{i=0}^{n-\alpha} \int_{M} \hat{u} \chi_{\hat{u}}^{i} \wedge \chi^{n-\alpha-i} \wedge \omega^{\alpha}=0 .
$$

The first inequality in (4.20) then follows from (4.22).

Rewriting (4.22),

$$
\int_{M} \hat{u} \chi^{n-\alpha} \wedge \omega^{\alpha}=-\sum_{i=1}^{n-\alpha} \int_{M} \hat{u} \chi_{\hat{u}}^{i} \wedge \chi^{n-\alpha-i} \wedge \omega^{\alpha}
$$

Let $C_{1}$ be a positive constant such that

$$
\omega^{n} \leq C_{1} \chi^{n-\alpha} \wedge \omega^{\alpha}
$$


Then

$$
\begin{aligned}
\int_{M} \hat{u} \omega^{n} & =\int_{M}\left(\hat{u}-\inf _{M} \hat{u}\right) \omega^{n}+\int_{M} \inf _{M} \hat{u} \omega^{n} \\
& \leq C_{1} \int_{M}\left(\hat{u}-\inf _{M} \hat{u}\right) \chi^{n-\alpha} \wedge \omega^{\alpha}+\inf _{M} \hat{u} \int_{M} \omega^{n} \\
\leq & -C_{1} \sum_{i=1}^{n-\alpha} \int_{M} \hat{u} \chi_{\hat{u}}^{i} \wedge \chi^{n-\alpha-i} \wedge \omega^{\alpha} \\
& \quad+\inf _{M} \hat{u}\left(\int_{M} \omega^{n}-C_{1} \int_{M} \chi^{n-\alpha} \wedge \omega^{\alpha}\right) \\
& =-C_{1} \sum_{i=1}^{n-\alpha} \int_{M}\left(\hat{u}-\inf _{M} \hat{u}\right) \chi_{\hat{u}}^{i} \wedge \chi^{n-\alpha-i} \wedge \omega^{\alpha} \\
& +\inf _{M} \hat{u}\left(\int_{M} \omega^{n}-(n-\alpha+1) C_{1} \int_{M} \chi^{n-\alpha} \wedge \omega^{\alpha}\right) \\
\leq & \inf _{M} \hat{u}\left(\int_{M} \omega^{n}-(n-\alpha+1) C_{1} \int_{M} \chi^{n-\alpha} \wedge \omega^{\alpha}\right) .
\end{aligned}
$$

The second inequality (4.20) then follows from (4.25), the fact that $\Delta_{\omega} u>-t_{\omega} \chi$ and the lower bound of the Green's function of $\omega$ (see Yau [27]).

It remains to prove Theorem 4.2.

Proof of Theorem 4.2. From Lemma 4.3 and the simple fact that

$$
\sup _{M} u(x, t)-\inf _{M} u(x, t)=\sup _{M} \hat{u}(x, t)-\inf _{M} \hat{u}(x, t),
$$

it suffices to prove a lower bound for $\inf _{M} \hat{u}(x, t)$. If such a lower bound does not exist, then we can choose a sequence of time points $t_{i} \rightarrow \infty$ such that

$$
\inf _{M} \hat{u}\left(x, t_{i}\right)=\inf _{t \in\left[0, t_{i}\right]} \inf _{M} \hat{u}(x, t)
$$

and

$$
\inf _{M} \hat{u}\left(x, t_{i}\right) \rightarrow-\infty \text {. }
$$

Then for any $t \leq t_{i}$,

$$
\inf _{M} u\left(x, t_{i}\right)-\inf _{M} u(x, t) \leq \frac{J_{\alpha}\left(u\left(t_{i}\right)\right)}{\int_{M} \chi^{n-\alpha} \wedge \omega^{\alpha}}-\frac{J_{\alpha}(u(t))}{\int_{M} \chi^{n-\alpha} \wedge \omega^{\alpha}} \leq 0 .
$$


With a similar argument, we also obtain

$$
u(x, t) \leq \hat{u}(x, t) .
$$

So we have

$$
\inf _{M} u\left(x, t_{i}\right)=\inf _{t \in\left[0, t_{i}\right]} \inf _{M} u(x, t)
$$

and

$$
\inf _{M} u\left(x, t_{i}\right) \rightarrow-\infty .
$$

By Proposition 3.1,

$$
\begin{aligned}
w\left(x, t_{i}\right) & \leq C e^{A\left(u\left(x, t_{i}\right)-\inf _{M \times\left[0, t_{i}\right]} u\right)} \\
& =C e^{A\left(u\left(x, t_{i}\right)-\inf _{M} u\left(x, t_{i}\right)\right)} .
\end{aligned}
$$

As shown in [23], the sharp $C^{2}$ estimate implies that

$$
\sup _{M} \hat{u}\left(x, t_{i}\right)-\inf _{M} \hat{u}\left(x, t_{i}\right)=\sup _{M} u\left(x, t_{i}\right)-\inf _{M} u\left(x, t_{i}\right) \leq C,
$$

for some positive constant $C$, which is a contradiction since $\sup _{M} \hat{u}\left(x, t_{i}\right)$ is bounded from below by zero.

For some $t_{0} \in[0, t], \inf _{M} u\left(x, t_{0}\right)=\inf _{M \times[0, t]} u(x, s)$ and hence

$$
\begin{aligned}
u(x, t)-\inf _{M \times[0, t]} u(x, s) & =u(x, t)-\inf _{M} u\left(x, t_{0}\right) \\
& =\hat{u}(x, t)-\inf _{M} u\left(x, t_{0}\right)+\frac{J_{\alpha}(u(t))}{\int_{M} \chi^{n-\alpha} \wedge \omega^{\alpha}} \\
& \leq \hat{u}(x, t)-\inf _{M} \hat{u}\left(x, t_{0}\right) .
\end{aligned}
$$

The proof of Theorem 4.2 tells us that $\hat{u}(x, t)$ is uniformly bounded on $M \times[0, \infty)$. Consequently,

$$
w(x, t) \leq C e^{A\left(\sup _{M} \hat{u}(x, t)-\inf _{M} \hat{u}\left(x, t_{0}\right)\right)}<C .
$$

Now we should use Evan-Krylov theory and Schauder estimate again to obtain uniform higher order estimates. 


\section{The Harnack Inequality}

In this section, we prove the Harnack inequality. The arguments of Gill [15] can be applied here. For completeness, we include the proof.

Set $F(u):=\frac{S_{n}\left(\chi_{u}\right)}{S_{n-\alpha}\left(\chi_{u}\right)}$. We define a new Hermitian metric corresponding to $u$,

$$
G:=F(u) \sum_{i, j} F_{i \bar{j}}(u) d z^{i} \wedge d \bar{z}^{j}
$$

and thus

$$
\left\{\begin{array}{l}
G_{i \bar{j}}=F(u) F_{i \bar{j}}(u), \\
G^{i \bar{j}}=F^{-1}(u) F^{i \bar{j}}
\end{array}\right.
$$

where $F^{i \bar{j}}:=\frac{\partial F}{\partial u_{i \bar{j}}}$ and $\left[F_{i \bar{j}}\right]_{n \times n}$ is the inverse of $\left[F^{i \bar{j}}\right]_{n \times n}$.

Constructing the Hermitian metric is a key technique in [21] to carry out method of continuity, while the metric is quite natural in the study of the parabolic flow, especially when we study the properties of higher order derivatives, such as the Harnack inequality, Evans-Krylov theory and Schauder estimate.

Let $\varphi$ be a positive function on $M$. We consider the linearized parabolic equation

$$
\partial_{t} \varphi=\sum_{i, j} G^{i \bar{j}} \bar{\partial}_{j} \partial_{i} \varphi
$$

Define $f=\ln \varphi$ and $H=t\left(|\partial f|_{G}^{2}-\beta \partial_{t} f\right)$ where $1<\beta<2$. Here

$$
|\partial f|_{G}^{2}=G(\partial f, \bar{\partial} f)=\sum_{i, j} G^{i \bar{j}} \partial_{i} f \bar{\partial}_{j} f .
$$

We also denote

$$
\langle X, Y\rangle_{G}=G(X, \bar{Y})=\sum_{i, j} G^{i \bar{j}} X_{i} Y_{\bar{j}}
$$

Then

$$
\begin{aligned}
& \partial_{t} f-\sum_{i, j} G^{i \bar{j}} \bar{\partial}_{j} \partial_{i} f \\
= & \frac{\partial_{t} \varphi}{\varphi}-\frac{1}{\varphi} \sum_{i, j} G^{i \bar{j}} \bar{\partial}_{j} \partial_{i} \varphi+\frac{1}{\varphi^{2}} \sum_{i, j} G^{i \bar{j}} \partial_{i} f \bar{\partial}_{j} f \\
= & |\partial f|_{G}^{2} .
\end{aligned}
$$


Lemma 5.1. There are constants $C_{1}$ and $C_{2}$ depending only on the bounds of the metric $G$ such that for $t>0$, we have

$$
\begin{aligned}
\partial_{t} H-\sum_{k, l} G^{k \bar{l}} \bar{\partial}_{l} \partial_{k} H \leq & -\frac{t}{2 n}\left(|\partial f|_{G}^{2}-\partial_{t} f\right)^{2}+2 \mathfrak{R e}\langle\partial f, \partial H\rangle_{G} \\
& +\left(|\partial f|_{G}^{2}-\beta \partial_{t} f\right)+C_{1} t|\partial f|_{G}^{2}+C_{2} t
\end{aligned}
$$

Proof. By (5.6),

$$
\begin{aligned}
H & =t\left(|\partial f|_{G}^{2}-\beta \partial_{t} f\right) \\
& =t \partial_{t} f-t \sum_{i, j} G^{i \bar{j}} \bar{\partial}_{j} \partial_{i} f-t \beta \partial_{t} f \\
& =-t \sum_{i, j} G^{i \bar{j}} \bar{\partial}_{j} \partial_{i} f-t(\beta-1) \partial_{t} f .
\end{aligned}
$$

Then,

$$
\sum_{i, j} G^{i \bar{j}} \bar{\partial}_{j} \partial_{i} f=-\frac{1}{t} H-(\beta-1) \partial_{t} f
$$

and consequently

$$
\frac{\partial}{\partial t}\left(\sum_{i, j} G^{i \bar{j}} \bar{\partial}_{j} \partial_{i} f\right)=\frac{1}{t^{2}} H-\frac{1}{t} \partial_{t} H-(\beta-1) \partial_{t}^{2} f
$$

Direct calculation shows that

$$
\begin{aligned}
\partial_{t} H & =|\partial f|_{G}^{2}-\beta \partial_{t} f+t \partial_{t}\left(\sum_{i, j} G^{i \bar{j}} \partial_{i} f \bar{\partial}_{j} f\right)-\beta t \partial_{t}^{2} f \\
& =|\partial f|_{G}^{2}-\beta \partial_{t} f+t \sum_{i, j} \partial_{t} G^{i \bar{j}} \partial_{i} f \bar{\partial}_{j} f+2 t \Re \mathfrak{k}\left\langle\partial f, \partial \partial_{t} f\right\rangle_{G}-\beta t \partial_{t}^{2} f
\end{aligned}
$$

and hence

$$
-2 \mathfrak{R e}\left\langle\partial f, \partial \partial_{t} f\right\rangle_{G}=-\frac{1}{t} \partial_{t} H+\frac{1}{t}|\partial f|_{G}^{2}-\frac{\beta}{t} \partial_{t} f+\sum_{i, j} \partial_{t} G^{i \bar{j}} \partial_{i} f \bar{\partial}_{j} f-\beta \partial_{t}^{2} f
$$


Also,

$$
\begin{aligned}
\sum_{k, l} G^{k \bar{l}} \bar{\partial}_{l} \partial_{k} H & =t \sum_{k, l} G^{k \bar{l}}\left(\sum_{i, j} \bar{\partial}_{l} \partial_{k} G^{i \bar{j}} \partial_{i} f \bar{\partial}_{j} f+\sum_{i, j} \partial_{k} G^{i \bar{j}} \bar{\partial}_{l} \partial_{i} f \bar{\partial}_{j} f\right. \\
& +\sum_{i, j} \partial_{k} G^{i \bar{j}} \partial_{i} f \bar{\partial}_{l} \bar{\partial}_{j} f+\sum_{i, j} \bar{\partial}_{l} G^{i \bar{j}} \partial_{k} \partial_{i} f \bar{\partial}_{j} f+\sum_{i, j} \bar{\partial}_{l} G^{i \bar{j}} \partial_{i} f \partial_{k} \bar{\partial}_{j} f \\
& +\sum_{i, j} G^{i \bar{j}} \bar{\partial}_{l} \partial_{k} \partial_{i} f \bar{\partial}_{j} f+\sum_{i, j} G^{i \bar{j}} \partial_{k} \partial_{i} f \bar{\partial}_{l} \bar{\partial}_{j} f+\sum_{i, j} G^{i \bar{j}} \bar{\partial}_{l} \partial_{i} f \partial_{k} \bar{\partial}_{j} f \\
& \left.+\sum_{i, j} G^{i \bar{j}} \partial_{i} f \bar{\partial}_{l} \partial_{k} \bar{\partial}_{j} f-\beta \bar{\partial}_{l} \partial_{k} \partial_{t} f\right)
\end{aligned}
$$

Now we control all the terms in $(5.13)$ by the bounds obtained in the previous sections. We bound the first term by

$$
\left|\sum_{i, j, k, l} G^{k \bar{l}} \bar{\partial}_{l} \partial_{k} G^{i \bar{j}} \partial_{i} f \bar{\partial}_{j} f\right| \leq C_{1}|\partial f|_{G}^{2}
$$

We bound the second, third, fourth and fifth terms by

$$
\begin{aligned}
& \left|\sum_{i, j, k, l} G^{k \bar{l}} \partial_{k} G^{i \bar{j}} \bar{\partial}_{l} \partial_{i} f \bar{\partial}_{j} f\right| \leq \frac{C_{2}}{\epsilon}|\partial f|_{G}^{2}+\epsilon|\partial \bar{\partial} f|_{G}^{2}, \\
& \left|\sum_{i, j, k, l} G^{k \bar{l}} \partial_{k} G^{i \bar{j}} \partial_{i} f \bar{\partial}_{l} \bar{\partial}_{j} f\right| \leq \frac{C_{2}}{\epsilon}|\partial f|_{G}^{2}+\epsilon\left|D^{2} f\right|_{G}^{2}, \\
& \left|\sum_{i, j, k, l} G^{k \bar{l}} \bar{\partial}_{l} G^{i \bar{j}} \partial_{k} \partial_{i} f \bar{\partial}_{j} f\right| \leq \frac{C_{2}}{\epsilon}|\partial f|_{G}^{2}+\epsilon|\partial \bar{\partial} f|_{G}^{2},
\end{aligned}
$$

and

$$
\left|\sum_{i, j, k, l} G^{k \bar{l}} \bar{\partial}_{l} G^{i \bar{j}} \partial_{i} f \partial_{k} \bar{\partial}_{j} f\right| \leq \frac{C_{2}}{\epsilon}|\partial f|_{G}^{2}+\epsilon\left|D^{2} f\right|_{G}^{2}
$$

where

$$
|\partial \bar{\partial} f|_{G}^{2}=\sum_{i, j, k, l} G^{k \bar{l}} G^{i \bar{j}} \bar{\partial}_{l} \partial_{i} f \partial_{k} \bar{\partial}_{j} f, \quad\left|D^{2} f\right|_{G}^{2}=\sum_{i, j, k, l} G^{k \bar{l}} G^{i \bar{j}} \partial_{k} \partial_{i} f \bar{\partial}_{l} \bar{\partial}_{j} f .
$$

It is obvious that the seventh and eighth terms are

$$
\sum_{i, j, k, l} G^{k \bar{l}} G^{i \bar{j}} \partial_{k} \partial_{i} f \bar{\partial}_{l} \bar{\partial}_{j} f=\left|D^{2} f\right|_{G}^{2}
$$


and

$$
\sum_{i, j, k, l} G^{k \bar{l}} G^{i \bar{j}} \bar{\partial}_{l} \partial_{i} f \partial_{k} \bar{\partial}_{j} f=|\partial \bar{\partial} f|_{G}^{2}
$$

From (5.9) and (5.12), the sixth and nineth terms together give

$$
\begin{aligned}
& \sum_{i, j, k, l} G^{k \bar{l}} G^{i \bar{j}}\left(\bar{\partial}_{l} \partial_{k} \partial_{i} f \bar{\partial}_{j} f+\partial_{i} f \bar{\partial}_{l} \partial_{k} \bar{\partial}_{j} f\right) \\
& \geq 2 \mathfrak{R e}\left\langle\partial f, \partial\left(\sum_{k, l} G^{k \bar{l}} \bar{\partial}_{l} \partial_{k} f\right)\right\rangle_{G}-\frac{C_{3}}{\epsilon}|\partial f|_{G}^{2}-\epsilon|\partial \bar{\partial} f|_{G}^{2} \\
& =-\frac{2}{t} \mathfrak{R e}\langle\partial f, \partial H\rangle_{G}-\frac{\beta-1}{t} \partial_{t} H+\frac{\beta-1}{t}|\partial f|_{G}^{2}-\frac{\beta(\beta-1)}{t} \partial_{t} f \\
& \quad+(\beta-1) \sum_{i, j} \partial_{t} G^{i \bar{j}} \partial_{i} f \bar{\partial}_{j} f-\beta(\beta-1) \partial_{t}^{2} f-\frac{C_{3}}{\epsilon}|\partial f|_{G}^{2}-\epsilon|\partial \bar{\partial} f|_{G}^{2} \\
& \geq-\frac{2}{t} \mathfrak{R e}\langle\partial f, \partial H\rangle_{G}-\frac{\beta-1}{t} \partial_{t} H+\frac{\beta-1}{t}|\partial f|_{G}^{2}-\frac{\beta(\beta-1)}{t} \partial_{t} f \\
& \quad-C_{4}|\partial f|_{G}^{2}-\beta(\beta-1) \partial_{t}^{2} f-\frac{C_{3}}{\epsilon}|\partial f|_{G}^{2}-\epsilon|\partial \bar{\partial} f|_{G}^{2} .
\end{aligned}
$$

By (5.10), the last term gives

$$
\begin{aligned}
-\beta \sum_{k, l} G^{k \bar{l}} \bar{\partial}_{l} \partial_{k} \partial_{t} f & =\beta \sum_{k, l} \partial_{t} G^{k \bar{l}} \bar{\partial}_{l} \partial_{k} f-\beta \frac{\partial}{\partial t}\left(\sum_{k, l} G^{k \bar{l}} \bar{\partial}_{l} \partial_{k} f\right) \\
& \geq-\frac{C_{5}}{\epsilon}-\epsilon|\partial \bar{\partial} f|_{G}^{2}-\frac{\beta}{t^{2}} H+\frac{\beta}{t} \partial_{t} H+\beta(\beta-1) \partial_{t}^{2} f .
\end{aligned}
$$

Substituting(5.14) - (5.18), (5.20) - (5.23) into (5.13), we have

$$
\begin{aligned}
\sum_{k, l} G^{k \bar{l}} \bar{\partial}_{l} \partial_{k} H \geq & \partial_{t} H-2 \mathfrak{R e}\langle\partial f, \partial H\rangle_{G}-\left(|\partial f|_{G}^{2}-\beta \partial_{t} f\right)+t(1-4 \epsilon)|\partial \bar{\partial} f|_{G}^{2} \\
& -t\left(C_{1}+C_{4}+\frac{4 C_{2}}{\epsilon}+\frac{C_{3}}{\epsilon}\right)|\partial f|_{G}^{2}+t(1-2 \epsilon)\left|D^{2} f\right|_{G}^{2}-\frac{t C_{5}}{\epsilon} \\
\geq & \partial_{t} H-2 \mathfrak{R e}\langle\partial f, \partial H\rangle_{G}-\left(|\partial f|_{G}^{2}-\beta \partial_{t} f\right)+\frac{t}{2}|\partial \bar{\partial} f|_{G}^{2} \\
& -C t|\partial f|_{G}^{2}-C t
\end{aligned}
$$

when $\epsilon>0$ is small enough. Applying the Schwarz inequality,

$$
|\partial \bar{\partial} f|_{G}^{2} \geq \frac{\left(\sum_{k, l} G^{k \bar{l}} \bar{\partial}_{l} \partial_{k} f\right)^{2}}{n}=\frac{\left(\partial_{t} f-|\partial f|_{G}^{2}\right)^{2}}{n} \text {. }
$$


Therefore,

$$
\begin{aligned}
\sum_{k, l} G^{k \bar{l}} \bar{\partial}_{l} \partial_{k} H-\partial_{t} H \geq & -2 \mathfrak{R e}\langle\partial f, \partial H\rangle_{G}-\left(|\partial f|_{G}^{2}-\beta \partial_{t} f\right) \\
& +\frac{t}{2 n}\left(\partial_{t} f-|\partial f|_{G}^{2}\right)^{2}-C t|\partial f|_{G}^{2}-C t .
\end{aligned}
$$

Lemma 5.2. There exist uniform constants $C_{1}$ and $C_{2}$ such that for all $t>0$,

$$
|\partial f|_{G}^{2}-\beta \partial_{t} f \leq C_{1}+\frac{C_{2}}{t} \text {. }
$$

Proof. Fix $T>0$ and suppose that $H$ attains its maximum at $\left(p, t_{0}\right)$ in $M \times(0, T]$. Then at the point $\left(p, t_{0}\right)$,

$$
0 \geq-\left(|\partial f|_{G}^{2}-\beta \partial_{t} f\right)+\frac{t_{0}}{2 n}\left(\partial_{t} f-|\partial f|_{G}^{2}\right)^{2}-C_{1} t_{0}|\partial f|_{G}^{2}-C_{2} t_{0} .
$$

If $\partial_{t} f\left(p, t_{0}\right) \geq 0$,

$$
\begin{aligned}
0 & \geq-\left(|\partial f|_{G}^{2}-\partial_{t} f\right)+\frac{t_{0}}{2 n}\left(\partial_{t} f-|\partial f|_{G}^{2}\right)^{2}-C_{1} t_{0}|\partial f|_{G}^{2}-C_{2} t_{0} \\
& =\frac{t_{0}}{2 n}\left(|\partial f|_{G}^{2}-\partial_{t} f\right)\left(\partial_{t} f-|\partial f|_{G}^{2}-\frac{2 n}{t_{0}}\right)-C_{1} t_{0}|\partial f|_{G}^{2}-C_{2} t_{0} .
\end{aligned}
$$

Hence,

$$
|\partial f|_{G}^{2}-\partial_{t} f \leq C_{3}|\partial f|_{G}+C_{4}+\frac{C_{5}}{t_{0}} \leq\left(1-\frac{1}{\beta}\right)|\partial f|_{G}^{2}+C_{6}+\frac{C_{5}}{t_{0}}
$$

That is,

$$
|\partial f|_{G}^{2}-\beta \partial_{t} f \leq C_{7}+\frac{C_{8}}{t_{0}}
$$

If $\partial_{t} f\left(p, t_{0}\right)<0$,

$$
\begin{aligned}
0 & \geq-\left(|\partial f|_{G}^{2}-\beta \partial_{t} f\right)+\frac{t_{0}}{2 n}\left(\partial_{t} f-|\partial f|_{G}^{2}\right)^{2}-C_{1} t_{0}|\partial f|_{G}^{2}-C_{2} t_{0} \\
& \geq-|\partial f|_{G}^{2}+\beta \partial_{t} f+\frac{t_{0}}{2 n}|\partial f|_{G}^{4}-C_{1} t_{0}|\partial f|_{G}^{2}-C_{2} t_{0} \\
& =|\partial f|_{G}^{2}\left(-1-C_{1} t_{0}+\frac{t_{0}}{2 n}|\partial f|_{G}^{2}\right)+\beta \partial_{t} f-C_{2} t_{0} .
\end{aligned}
$$

That is,

$$
|\partial f|_{G}^{2}\left(-\frac{1}{t_{0}}-C_{1}+\frac{1}{2 n}|\partial f|_{G}^{2}\right) \leq-\frac{\beta}{t_{0}} \partial_{t} f+C_{2} .
$$


Hence,

$$
|\partial f|_{G}^{2} \leq C_{3}+\frac{C_{4}}{t_{0}}-\frac{1}{2} \partial_{t} f
$$

So

$$
\begin{aligned}
0 & \geq-\left(|\partial f|_{G}^{2}-\beta \partial_{t} f\right)+\frac{t_{0}}{2 n}\left(\partial_{t} f-|\partial f|_{G}^{2}\right)^{2}-C_{1} t_{0}|\partial f|_{G}^{2}-C_{2} t_{0} \\
& \geq-|\partial f|_{G}^{2}+\beta \partial_{t} f+\frac{t_{0}}{2 n}\left(\partial_{t} f\right)^{2}-C_{1} t_{0}|\partial f|_{G}^{2}-C_{2} t_{0} .
\end{aligned}
$$

By factoring the terms, we obtain

$$
\left(-\partial_{t} f\right)\left(-\frac{\beta}{t_{0}}-\frac{1}{2 n} \partial_{t} f\right) \leq \frac{1}{t_{0}}|\partial f|_{G}^{2}+C_{1}|\partial f|_{G}^{2}+C_{2} .
$$

Thus

$$
\begin{aligned}
-\partial_{t} f & \leq C_{5}+\frac{C_{6}}{t_{0}}+\frac{1}{2}|\partial f|_{G}^{2} \\
& \leq C_{5}+\frac{C_{6}}{t_{0}}+\frac{C_{3}}{2}+\frac{C_{4}}{2 t_{0}}-\frac{1}{4} \partial_{t} f
\end{aligned}
$$

and hence

$$
-\partial_{t} f \leq C_{7}+\frac{C_{8}}{t_{0}}
$$

Similarly

$$
\begin{aligned}
|\partial f|_{G}^{2} & \leq C_{3}+\frac{C_{4}}{t_{0}}-\frac{1}{2} \partial_{t} f \\
& \leq C_{3}+\frac{C_{4}}{t_{0}}+\frac{C_{5}}{2}+\frac{C_{6}}{2 t_{0}}+\frac{1}{4}|\partial f|_{G}^{2}
\end{aligned}
$$

and thus

$$
|\partial f|_{G}^{2} \leq C_{9}+\frac{C_{10}}{t_{0}}
$$

Combining (5.38) and (5.40),

$$
|\partial f|_{G}^{2}-\beta \partial_{t} f \leq C_{11}+\frac{C_{12}}{t_{0}} .
$$

Therefore,

$$
\begin{aligned}
H(x, T) & \leq H\left(p, t_{0}\right) \\
& =t_{0}\left(|\partial f|_{G}^{2}\left(p, t_{0}\right)-\beta \partial_{t} f\left(p, t_{0}\right)\right) \\
& \leq \beta\left(C_{1} t_{0}+C_{2}\right) \\
& \leq \beta\left(C_{1} T+C_{2}\right) .
\end{aligned}
$$


Lemma 5.3. $\forall 0<t_{1}<t_{2}$,

$$
\sup _{x \in M} \varphi\left(x, t_{1}\right) \leq \inf _{x \in M} \varphi\left(x, t_{2}\right)\left(\frac{t_{2}}{t_{1}}\right)^{C_{2}} e^{\frac{C_{3}}{t_{2}-t_{1}}+C_{1}\left(t_{2}-t_{1}\right)}
$$

where $C_{1}, C_{2}$ and $C_{3}$ are uniform constants.

Proof. Let $x, y \in M$, and define $\gamma$ to be the minimal geodesic (with respect ot the initial metric $\omega)$ with $\gamma(0)=y$ and $\gamma(1)=x$. Define a path $\zeta:[0,1] \rightarrow M \times\left[t_{1}, t_{2}\right]$ by $\zeta(s)=\left(\gamma(s),(1-s) t_{2}+s t_{1}\right)$. Then using Lemma 5.2 ,

$$
\begin{aligned}
\ln \frac{\varphi\left(x_{1}, t_{1}\right)}{\varphi\left(y_{2}, t_{2}\right)} & =\int_{0}^{1} \frac{d}{d s} f(\zeta(s)) d s \\
& =\int_{0}^{1}\left[2 \Re \mathfrak{R e}\langle\dot{\gamma}, \partial f\rangle-\left(t_{2}-t_{1}\right) \partial_{t} f\right] d s \\
& \leq \int_{0}^{1}\left[2|\dot{\gamma}|_{G}|\partial f|_{G}+\left(t_{2}-t_{1}\right)\left(C_{1}+\frac{C_{2}}{t}-\frac{1}{\beta}|\partial f|_{G}^{2}\right)\right] d s \\
& \leq \int_{0}^{1}\left[-\frac{t_{2}-t_{1}}{\beta}\left(|\partial f|_{G}-\frac{\beta|\dot{\gamma}|_{G}}{t_{2}-t_{1}}\right)^{2}+\frac{\beta|\dot{\gamma}|_{G}^{2}}{t_{2}-t_{1}}\right. \\
& \leq \int_{0}^{1}\left[\frac{C_{3}}{t_{2}-t_{1}}+C_{1}\left(t_{2}-t_{1}\right)+C_{2} \frac{t_{2}-t_{1}}{t}\right] d s \\
& =\frac{C_{3}}{t_{2}-t_{1}}+C_{1}\left(t_{2}-t_{1}\right)+C_{2} \ln \frac{t_{2}}{t_{1}} .
\end{aligned}
$$

\section{Convergence of the Parabolic Flow}

With the Harnack inequality, we can show the convergence of $\tilde{u}$ following Cao [5].

Define $\varphi=\frac{\partial u}{\partial t}$. Then we have

$$
\frac{\partial \varphi}{\partial t}=\sum_{i, j} G^{i \bar{j}} \bar{\partial}_{j} \partial_{i} \varphi
$$


Let $m$ be a positive integer and define

$$
\begin{aligned}
& \xi_{m}(x, t)=\sup _{y \in M} \varphi(y, m-1)-\varphi(x, m-1+t) \geq 0, \\
& \eta_{m}(x, t)=\varphi(x, m-1+t)-\inf _{y \in M} \varphi(y, m-1) \geq 0 .
\end{aligned}
$$

These functions satisfy the heat equations

$$
\begin{aligned}
\frac{\partial \xi_{m}}{\partial t} & =\sum_{i, j} G^{i \bar{j}}(m-1+t) \bar{\partial}_{j} \partial_{i} \xi_{m}, \\
\frac{\partial \eta_{m}}{\partial t} & =\sum_{i, j} G^{i \bar{j}}(m-1+t) \bar{\partial}_{j} \partial_{i} \eta_{m} .
\end{aligned}
$$

If $\varphi(x, m-1)$ is not a constant function, then $\xi_{m}>0$ for some $x \in M$ at time $t=0$. By the maximum principle, $\xi_{m}$ has to be positive for any $x \in M$ when $t>0$. Similarly, $\eta_{m}$ is also positive everywhere in $M \times(0, \infty)$. Hence, we can apply Lemma 5.3 with $t_{1}=\frac{1}{2}$ and $t_{2}=1$,

$$
\begin{aligned}
& \sup _{M} \varphi(x, m-1)-\inf _{M} \varphi\left(x, m-\frac{1}{2}\right) \leq C\left(\sup _{M} \varphi(x, m-1)-\sup _{M} \varphi(x, m)\right), \\
& \sup _{M} \varphi\left(x, m-\frac{1}{2}\right)-\inf _{M} \varphi(x, m-1) \leq C\left(\inf _{M} \varphi(x, m)-\inf _{M} \varphi(x, m-1)\right) .
\end{aligned}
$$

Define the oscillation $\theta(t)=\sup _{x \in M} \varphi(x, t)-\inf _{x \in M} \varphi(x, t)$. Adding the above inequalities gives us

$$
\theta(m-1)+\theta\left(m-\frac{1}{2}\right) \leq C(\theta(m-1)-\theta(m)) .
$$

So

$$
\theta(m) \leq \frac{C-1}{C} \theta(m-1)
$$

By induction, we have

$$
\theta(t) \leq C e^{-c_{0} t}
$$

where $c_{0}=-\ln \frac{C-1}{C}$. On the other hand, if $\varphi(x, m-1)$ is constant, this inequality still holds true since $\varphi(x, t)$ is then a constant function.

Since the normalized solution is defined by

$$
\tilde{u}=u-\frac{\int_{M} u \omega^{n}}{\int \omega^{n}}
$$


it is obvious that

$$
\int_{M} \frac{\partial \tilde{u}}{\partial t} \omega^{n}=0
$$

Fixing $(x, t)$ in $M \times[0, \infty)$, there is a point $y \in M$ such that

$$
\frac{\partial \tilde{u}}{\partial t}(y, t)=0 \text {. }
$$

Hence,

$$
\begin{aligned}
\left|\frac{\partial \tilde{u}}{\partial t}(x, t)\right| & =\left|\frac{\partial \tilde{u}}{\partial t}(x, t)-\frac{\partial \tilde{u}}{\partial t}(y, t)\right| \\
& =\left|\frac{\partial u}{\partial t}(x, t)-\frac{\partial u}{\partial t}(y, t)\right| \\
& \leq C e^{-c_{0} t} .
\end{aligned}
$$

Consider the quantity $Q=\tilde{u}+\frac{C}{c_{0}} e^{-c_{0} t}$,

$$
\frac{\partial Q}{\partial t} \leq 0
$$

Since $Q$ is bounded and pointwise monotonically decreasing, it tends to a limit as $t \rightarrow \infty$, say, $\tilde{u}_{\infty}$. But

$$
\lim _{t \rightarrow \infty} \tilde{u}=\lim _{t \rightarrow \infty} Q=\tilde{u}_{\infty}
$$

We show that the convergence of $\tilde{u}$ to $\tilde{u}_{\infty}$ is actually $C^{\infty}$ by contradiction. Suppose that the convergence is not $C^{\infty}$, then there exists a time sequence $t_{i} \rightarrow \infty$ such that for some $\epsilon>0$ and some integer $k$,

$$
\left\|\tilde{u}\left(x, t_{i}\right)-\tilde{u}_{\infty}\right\|_{C^{k}}>\epsilon, \quad \forall i .
$$

Since $\tilde{u}$ is bounded in $C^{\infty}$, there exists a subsequence $t_{i_{j}} \rightarrow \infty$ such that $\tilde{u}\left(x, t_{i_{j}}\right) \rightarrow$ $\tilde{U}_{\infty}$ as $j \rightarrow \infty$ for some smooth function $\tilde{U}_{\infty}$. Definitely, $\tilde{u}_{\infty} \neq \tilde{U}_{\infty}$. But it contradicts the fact that $\tilde{u} \rightarrow \tilde{u}_{\infty}$ pointwise.

Note that $\tilde{u}$ solves the parabolic flow

$$
\frac{\partial \tilde{u}}{\partial t}=\ln \frac{\chi_{\tilde{u}}^{n}}{\chi_{\tilde{u}}^{n-\alpha} \wedge \omega^{\alpha}}-\ln \psi-\frac{\int_{M} u \omega^{n}}{\int \omega^{n}} .
$$

Letting $t \rightarrow \infty, \tilde{u}_{\infty}$ solves the equation

$$
\ln \frac{\chi_{\tilde{u}}^{n}}{\chi_{\tilde{u}}^{n-\alpha} \wedge \omega^{\alpha}}=\ln \psi+b
$$


where

$$
b=\frac{\int_{M}\left(\ln \frac{\chi_{\tilde{u}}^{n}}{\chi_{\tilde{u}}^{n-\alpha} \wedge \omega^{\alpha}}-\ln \psi\right) \omega^{n}}{\int_{M} \omega^{n}}=\lim _{t \rightarrow \infty} \frac{\int_{M}\left(\ln \frac{\chi_{u}^{n}}{\chi_{u}^{n-\alpha} \wedge \omega^{\alpha}}-\ln \psi\right) \omega^{n}}{\int_{M} \omega^{n}} .
$$

This completes the proof the convergence.

\section{REVISIT TO METHOD OF CONTINUITY}

In this section, we want to avoid using the specific knowledge of $J$-functionals, Gauduchon's theorem [14] and Buchdahl's result [2]. So we apply the parabolic result, Theorem 1.2, to carrying out method of continuity.

Let us recall the a priori esimates for elliptic complex Monge-Ampère type equations in [21].

Theorem 7.1. . Let $\left(M^{n}, \omega\right)$ be a closed Hermitian manifold of complex dimension $n$ and $u$ be a smooth solution of the equaion (1.3). Suppose that $\chi \in \mathscr{C}_{\alpha}(\psi)$. Then there are uniform $C^{\infty}$ a priori estimates of $u$.

First, since $\chi \in \mathscr{C}_{\alpha}(\psi)$, there must be a function $\underline{u}$ satisfying

$$
\chi_{\underline{u}}=\chi+\frac{\sqrt{-1}}{2} \partial \bar{\partial} \underline{u}>0
$$

and

$$
n \chi_{\underline{u}}^{n-1}>(n-\alpha) \psi \chi_{\underline{u}}^{n-\alpha-1} \wedge \omega^{\alpha} .
$$

As in [21], without loss of generality, we can assume $\underline{u}$ is smooth.

Define $\underline{\varphi}$ by

$$
\chi_{\underline{u}}^{n}=\underline{\varphi} \chi_{\underline{u}}^{n-\alpha} \wedge \omega^{\alpha} .
$$

It follows from the monotonicity of $\frac{S_{n}}{S_{n-\alpha}}$ that

$$
n \chi_{\underline{u}}^{n-1}>(n-\alpha) \underline{\varphi} \chi_{\underline{u}}^{n-\alpha-1} \wedge \omega^{\alpha} .
$$

Consequently,

$$
n \chi_{\underline{u}}^{n-1}>(n-\alpha)(\max \{\psi, \underline{\varphi}\}+2 \delta) \chi_{\underline{u}}^{n-\alpha-1} \wedge \omega^{\alpha} .
$$

for sufficiently small $\delta>0$. By approximation, we can find a smooth function $\psi_{0}$ satisfying

$$
\max \{\psi, \underline{\varphi}\} \leq \psi_{0} \leq \max \{\psi, \underline{\varphi}\}+\delta
$$


So we have $\chi_{\underline{u}} \in \mathscr{C}_{\alpha}\left(\psi_{0}\right)$ and

$$
\frac{\chi_{\underline{u}}^{n}}{\chi_{\underline{u}}^{n-\alpha} \wedge \omega^{\alpha}} \leq \psi_{0}
$$

By Theorem 1.2 and maximum principle, there exists an admissible solution $u_{0}$ of

$$
\left(\chi+\frac{\sqrt{-1}}{2} \partial \bar{\partial} u\right)^{n}=\psi_{0} e^{b_{0}}\left(\chi+\frac{\sqrt{-1}}{2} \partial \bar{\partial} u\right)^{n-\alpha} \wedge \omega^{\alpha}
$$

for some $b_{0} \leq 0$.

Second, we start the method of continuity from $\chi_{u_{0}}$ and consider the family of equations

$$
\left(\chi+\frac{\sqrt{-1}}{2} \partial \bar{\partial} u_{s}\right)^{n}=\psi^{s} \psi_{0}^{1-s} e^{b_{s}}\left(\chi+\frac{\sqrt{-1}}{2} \partial \bar{\partial} u_{s}\right)^{n-\alpha} \wedge \omega^{\alpha}, \quad \text { for } s \in[0,1] .
$$

Note that $b_{0}$ has been found out in the first stage.

Integrating equation (7.9),

$$
\int_{M} \chi^{n}=\int_{M} \psi^{s} \psi_{0}^{1-s} e^{b_{s}}\left(\chi+\frac{\sqrt{-1}}{2} \partial \bar{\partial} u_{s}\right)^{n-\alpha} \wedge \omega^{\alpha} \geq c e^{b_{s}} \int_{M} \chi^{n-\alpha} \wedge \omega^{\alpha},
$$

which implies

$$
b_{s} \leq 0
$$

So we have

$$
\psi^{s} \psi_{0}^{1-s} e^{b_{s}} \leq \psi^{s} \psi_{0}^{1-s} \leq \psi_{0}
$$

Therefore, there are uniform $C^{\infty}$ estimates of $u_{s}$, as a consequnce of Theorem 7.1.

We consider the set

$$
\mathcal{S}:=\left\{s^{\prime} \in[0,1] \mid \exists u_{s} \in C^{2, \alpha}(M) \text { and } b_{s} \text { solving (7.9) for } s \in\left[0, s^{\prime}\right]\right\} .
$$

Since we obtained $b_{0}$ in the first stage, $0 \in \mathcal{S}$ and hence $\mathcal{S}$ is not empty. It suffices to show that $\mathcal{S}$ is both open and closed in $[0,1]$.

From equation (7.9), we have $\psi^{s} \psi_{0}^{1-s} e^{b_{s}} \geq \psi_{0} e^{b_{0}}$ when $u_{s}$ achieves its minimum. So

$$
0 \geq b_{s} \geq \inf _{M}\left(\ln \psi_{0}-\ln \psi\right)+b_{0} .
$$

The closedness of $\mathcal{S}$ follows from the uniform bound for $b_{s}$ and uniform $C^{\infty}$ estimates of $u_{s}$. 
Now we show that $\mathcal{S}$ is open. Assuming that $\hat{s} \in \mathcal{S}$, we need to show that there exists small $\epsilon>0$ such that $s \in \mathcal{S}$ for any $s \in[\hat{s}, \hat{s}+\epsilon)$.

$$
\left(\chi+\frac{\sqrt{-1}}{2} \partial \bar{\partial} u_{\hat{s}}\right)^{n}=\psi^{\hat{s}} \psi_{0}^{1-\hat{s}} e^{b_{\hat{s}}}\left(\chi+\frac{\sqrt{-1}}{2} \partial \bar{\partial} u_{\hat{s}}\right)^{n-\alpha} \wedge \omega^{\alpha} .
$$

When $\psi \equiv \psi_{0}$, the openess is obvious. If $\psi \not \equiv \psi_{0}$, we choose a positive constant $\kappa>1$ satisfying

$$
\kappa \psi_{0} \leq \psi_{0}+\delta
$$

Thus, we have

$$
\kappa \psi^{s} \psi_{0}^{1-s} \leq \psi_{0}+\delta
$$

and hence

$$
n \chi_{\underline{u}}^{n-1}>(n-\alpha) \kappa \psi^{s} \psi_{0}^{1-s} \chi_{\underline{u}}^{n-\alpha-1} \wedge \omega^{\alpha} .
$$

Also,

$$
\begin{aligned}
\kappa \psi^{s} \psi_{0}^{1-s} & \geq \kappa e^{-b_{\hat{s}}}\left(\frac{\psi}{\psi_{0}}\right)^{s-\hat{s}} \psi^{\hat{s}} \psi_{0}^{1-\hat{s}} e^{b_{\hat{s}}} \\
& \geq \kappa\left(\frac{\psi}{\psi_{0}}\right)^{s-\hat{s}} \psi^{\hat{s}} \psi_{0}^{1-\hat{s}} e^{b_{\hat{s}}} \\
& \geq \kappa\left(\inf _{M} \frac{\psi}{\psi_{0}}\right)^{s-\hat{s}} \psi^{\hat{s}} \psi_{0}^{1-\hat{s}} e^{b_{\hat{s}}} \\
& \geq \psi^{\hat{s}} \psi_{0}^{1-\hat{s}} e^{b_{\hat{s}}} \\
& =\frac{\chi_{u_{\hat{s}}}^{n}}{\chi_{u_{\hat{s}}^{n-\alpha}} \wedge \omega^{\alpha}}
\end{aligned}
$$

whenever

$$
s-\hat{s} \leq-\frac{\ln \kappa}{\ln \inf _{M} \frac{\psi}{\psi_{0}}} .
$$

Let $\epsilon=-\frac{\ln \kappa}{\inf _{M}\left(\ln \psi-\ln \psi_{0}\right)}$ and consider the parabolic equation

$$
\frac{\partial u}{\partial t}=\ln \frac{\chi_{u}^{n}}{\chi_{u}^{n-\alpha} \wedge \omega^{\alpha}}-\ln \left(\kappa \psi^{s} \psi_{0}^{1-s}\right),
$$

with $u(x, t)=u_{\hat{s}}(x)$. Therefore, from (7.18), (7.19) and Theorem 1.2, we have a pair $\left(u_{s}, b_{s}\right)$ solving equation (7.9) for $s \in[\hat{s}, \hat{s}+\epsilon)$, and hence $\mathcal{S}$ is open. 


\section{REFERENCES}

[1] T. Aubin, Équations du type Monge-Ampère sur les variétés kählériennes compactes, (French) Bull. Sci. Math. (2) 102 (1978), 63-95.

[2] N. Buchdahl, On compact Kähler surfaces, Ann. Inst. Fourier (Grenoble) 49 (1999), no. 1, 287-302.

[3] E. Calabi, The space of Kähler metrics, Proc. ICM, Amsterdam 1954, Vol. 2, 206-207, NorthHolland, Amsterdam, 1956.

[4] E. Calabi, On Kähler manifolds with vanishing canonical class, in Algebraic geometry and topology: A symposium in honor of S. Lefschetz, 78-89. Princeton University Press, 1957.

[5] H.-D. Cao, Deformation of Kähler metrics to Kähler-Einstein metrics on compact Khler manifolds, Invent. Math. 81 (1985), 359-372.

[6] X.-X. Chen On the lower bound of the Mabuchi energy and its application, Internat. Math. Res. Notices 2000, no. 12, 607-623

[7] X.-X. Chen, A new parabolic flow in Kähler manifolds, Comm. Anal. Geom. 12 (2004), 837-852.

[8] P. Cherrier, Equations de Monge-Ampère sur les variétés hermitiennes compactes, Bull. Sci. Math. 111 (1987), 343-385.

[9] S. K. Donaldson, Moment maps and diffeomorphisms, Asian J. Math. 3 (1999), 1-16.

[10] L. C. Evans, Classical solutions of fully nonlinear, convex, secondorder elliptic equations, Comm. Pure Appl. Math. 35 (1982), 333-363.

[11] H. Fang, M.-J. Lai and X.-N. Ma, On a class of fully nonlinear flows in Kähler geometry, J. Reine Angew. Math. 653 (2011), 189-220.

[12] H. Fang and M.-J. Lai, On the geometric flows solving Kählerian inverse $\sigma_{k}$ equations, Pacific J. Math. 258 (2012), no. 2, 291-304.

[13] H. Fang and M.-J. Lai, Convergence of general inverse $\sigma_{k}$-flow on Kähler manifolds with Calabi ansatz, Trans. Amer. Math. Soc. 365 (2013), no. 12, 6543-6567.

[14] P. Gauduchon, Le théorème de l'excentricité nulle, C. R. Acad. Sci. Paris 285 (1977), 387-390.

[15] M. Gill, Convergence of the parabolic complex Monge-Ampère equation on compact Hermitian manifolds, Comm. Anal. Geom. 19 (2011), no. 2, 277-304.

[16] B. Guan and Q. Li, Complex Monge-Ampère equations and totally real submanifolds, Adv. Math. 225 (2010) 1185-1223.

[17] B. Guan and W. Sun, On a class of fully nonlinear elliptic equations on Hermitian manifolds, to apprear in Calculus of Variations and PDE.

[18] N. V. Krylov, Boundedly nonhomogeneous elliptic and parabolic equations, Izvestiya Ross. Akad. Nauk. SSSR 46 (1982), 487-523.

[19] D. H. Phong and J. Sturm, The Dirichlet problem for degenerate complex Monge-Ampère equations, Comm. Anal. Geom. 18 (2010), no. 1, 145-170.

[20] J. Song and B. Weinkove, On the convergence and singularities of the J-flow with applications to the Mabuchi energy, Comm. Pure Appl. Math. 61 (2008), 210-229.

[21] W. Sun, On a class of fully nonlinear elliptic equations on closed Hermitian manifolds, preprint (2013).

[22] W. Sun, Parabolic complex Monge-Ampr̀e type equations on Hermtian manidolds, in preparation (2013).

[23] V. Tosatti and B. Weinkove, Estimates for the complex Monge-Ampère equation on Hermitian and balanced manifolds, Asian J. Math. 14 (2010), 19-40.

[24] V. Tosatti and B. Weinkove, The complex Monge-Ampère equation on compact Hermitian manifolds, J. Amer. Math. Soc. 23 (2010), 1187-1195. 
[25] B. Weinkove, Convergence of the J-flow on Kähler surfaces, Comm. Anal. Geom. 12 (2004), 949-965.

[26] B. Weinkove, On the J-flow in higher dimensions and the lower boundedness of the Mabuchi energy, J. Differential Geom. 73 (2006), no. 2, 351-358.

[27] S.-T. Yau, On the Ricci curvature of a compact Kähler manifold and the complex Monge-Ampère equation. I. Comm. Pure Appl. Math. 31 (1978), no. 3, 339-411.

Department of Mathematics, Ohio State University, Columbus, OH 43210

E-mail address: sun@math.ohio-state.edu 